\title{
Factors Related to Preoperative Shoulder Pain in Patients with Atraumatic Painful Rotator Cuff Tears
}

\author{
In Park, Hyo-Jin Lee ${ }^{1}$, Sang-Ki Kim², Min-Sik Park ${ }^{1}$, Yang-Soo Kim ${ }^{1 \Xi}$ \\ Department of Orthopedic Surgery, Ewha Womans University College of Medicine, ${ }^{1}$ Department of Orthopedic Surgery, Seoul St. Mary's Hospital, College of \\ Medicine, The Catholic University of Korea, Seoul, ${ }^{2}$ Yonsei Hangmac Surgical Clinic, Goyang, Korea
}

Background: Patients with rotator cuff tears are usually afflicted with shoulder pain and disability. However, it is unclear which factors are related to shoulder pain in patients with rotator cuff tears. This study was therefore undertaken to determine the factors correlated with shoulder pain in patients with painful rotator cuff tears, but without any history of trauma.

Methods: We evaluated a cohort of 745 patients with painful rotator cuff tears having no trauma history, and analyzed the relationship between pain and multiple factors including demographic data, tear characteristics, and passive range of motion. Pain was analyzed with a questionnaire concerning the visual analogue scale (VAS) for pain. Tear characteristics were determined by evaluating tear size, muscle atrophy, number of torn tendons, and presence of arthritis. Multivariate linear regression analysis and chi-squared test were applied to evaluate the relationship between the VAS for pain and variable factors.

Results: Shoulder pain was associated with young age $(p=0.01)$, male sex $(p=0.01)$ and the presence of diabetes mellitus $(p<0.001)$. Measurements of rotator cuff tear characteristics including tear size $(p=0.53)$, muscle atrophy $(p=0.16)$ and the number of torn tendons $(p=0.34)$ did not correlate with shoulder pain. Symptom duration $(p=0.60)$ and range of motion $(p>0.05)$ also showed no correlation with VAS for pain.

Conclusions: Young age, male sex and the presence of diabetes mellitus correlated positively with preoperative shoulder pain in patients with painful rotator cuff tears without a trauma history. Combined treatment of pain management and risk factor correction could be helpful to control preoperative shoulder pain.

(Clin Shoulder Elbow 2019;22(3):128-134)

Key Words: Rotator cuff; Pain; Risk factors; Sex; Diabetes mellitus

\section{Introduction}

Patients with rotator cuff tears usually complain of shoulder pain and disability. However, some patients have no pain despite rotator cuff tears. Recent articles report that these asymptomatic rotator cuff tears are found in $4.7 \%$ to $7.6 \%$ of the general population, and pain does not correlate with rotator cuff tear severity. ${ }^{1,2)}$ Asymptomatic rotator cuff tears frequently do not require operative treatment. Even in cases of painful full-thickness tears, conservative treatment is sometimes indicated despite torn tendons. $^{3,4)}$
Several previous studies have evaluated the factors correlated with pain in patients with rotator cuff tears. Harris et al. ${ }^{5}$ designed a cross-sectional study and concluded that the female sex, high education level and preserved shoulder strength significantly correlated with higher function and less pain. Disease severity is usually thought to be closely related to pain symptoms in many orthopedic diseases such as arthritis and synovitis. ${ }^{6,7)}$ Yamaguchi et al. ${ }^{8)}$ reported that the size of the rotator cuff tear might be an important predictor of pain. By contrast, several recent studies emphasized that symptoms do not predict severity of a rotator cuff tear. ${ }^{1,5,9)}$ In their cross-sectional study of 393

Received May 23, 2019. Revised July 29, 2019. Accepted July 31, 2019.

Correspondence to: Yang-Soo Kim

Department of Orthopedic Surgery, Seoul St. Mary's Hospital, The Catholic University of Korea, 222 Banpo-daero, Seocho-gu, Seoul 06591, Korea Tel: +82-2-2258-6117, Fax: +82-2-535-9834, E-mail: kysoos@catholic.ac.kr, ORCID: https://orcid.org/0000-0003-4267-7880

IRB approval: Seoul St. Mary's Hospital, The Catholic University of Korea (No. KC17RESI0120).

Financial support: None. Conflict of interests: None. 
patients, Dunn et al. ${ }^{1)}$ reported that pain from rotator cuff tear did not correlate with the tear severity measured by the number of torn tendons, amount of retraction, or degree of fatty degeneration. Wylie et al. ${ }^{9)}$ also reported that rotator cuff tear severity, including the tear size, do not correlate with pain or functional score, and pain is better correlated with mental health rather than tear size. However, these studies enrolled only full-thickness rotator cuff tears, and did not study partial-thickness tears. Partial-thickness tears, especially bursal-side partial-thickness tears, might be a positive factor for shoulder pain because of stress concentration in the remaining tendon. ${ }^{10,11)}$

Given the uncertain relationship between variable factors and shoulder pain, it is important to identify factors affecting shoulder pain in patients with rotator cuff tears. In the current study, we aimed to determine the factors correlated with shoulder pain in patients with painful rotator cuff tears, but with no history of trauma. We hypothesized that some patient-related factors, excluding tear characteristics, are associated with shoulder pain.

\section{Methods}

From December 2007 to May 2014, 1,379 patients who visited an outpatient clinic at a single hospital with painful rotator cuff tears were enrolled for the study. Inclusion criteria were partial-thickness or full-thickness painful rotator cuff tear confirmed by magnetic resonance imaging (MRI), positive physical examination including painful arc, and full can or empty can test. Exclusion criteria were patients with supraspinatus partial-thickness tear less than $50 \%$ of footprint or interstitial tear, symptomatic long head biceps tendon pathology, isolated subscapularis tendon tear, chief complaint of muscular pain such as myofascial pain syndrome or myositis, symptomatic cervical spine disease, a history of shoulder trauma or injection, bilateral rotator cuff tears, and history of previous shoulder surgery (Fig. 1).

All patients completed a questionnaire regarding the visual analogue scale (VAS) for pain and detailed demographic data including age, sex, symptom duration, and medical comorbidities (diabetes mellitus, hypertension, thyroid disease). The authors tried to evaluate pain that was restricted to the periarticular area (deltoid area pain); patients who mainly complained of neurologic radiating pain, anterior shoulder pain with bicipital groove tenderness, or muscular pain around trapezius muscle area rather than periarticular pain, were excluded from the present study. History of medical comorbidity was assessed by a simple questionnaire and chart review. Patients underwent a routine physical examination including painful arc test, full can and empty can test. The study protocol was approved by the Institutional Review Board of the Seoul St. Mary's Hospital, The Catholic University of Korea, and written informed consent was obtained from every patient.

A single musculoskeletal radiologist studied the MRI and plain

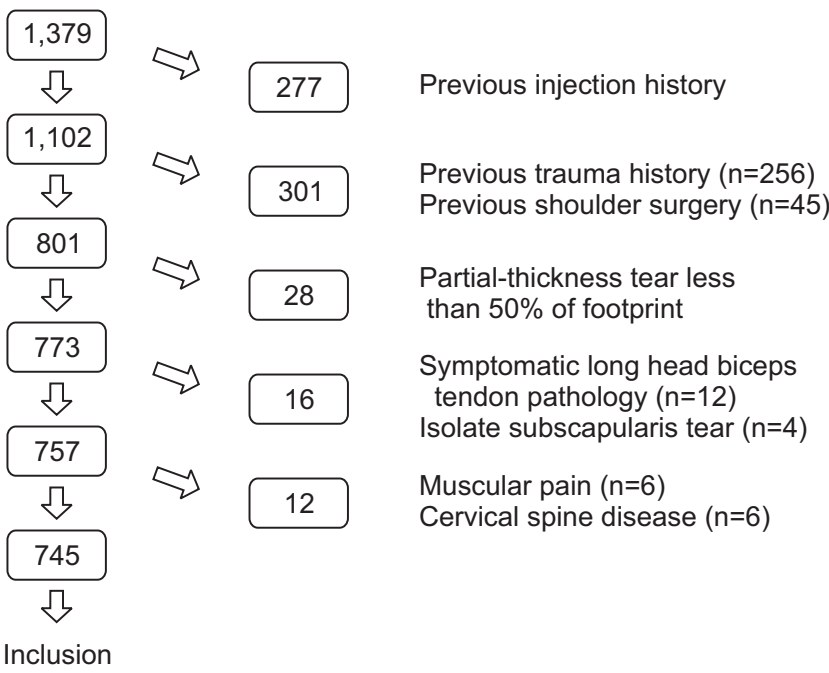

Fig. 1. Flowchart illustrating application of study inclusion and exclusion criteria.

radiographs, without knowledge of patient demographic data. Tear size, degree of supraspinatus muscle atrophy, number of torn tendons, glenohumeral joint arthritis, and acromioclavicular joint arthritis were recorded. Tear size was measured as the maximal distance between the lateral margin of the footprint and the torn tendon margin on T2-weighted coronal images, and categorized as small $(<1 \mathrm{~cm})$, medium $(1-3 \mathrm{~cm})$, large $(3-5$ $\mathrm{cm})$ or massive $(>5 \mathrm{~cm})$. Partial thickness tears were divided into bursal-side partial-thickness tear and articular-side partial-thickness tear. Muscle atrophy of the supraspinatus was measured by Goutallier's classification on T2-weighted sagittal images. ${ }^{12)}$ The number of torn tendons was measured by counting the number of full-thickness or partial-thickness tears of the supraspinatus, infraspinatus, subscapularis and teres minor tendon. Glenohumeral joint arthritis and acromioclavicular joint arthritis were appraised by the presence of joint space narrowing or osteophyte formation on plain radiographs (Kellgren-Lawrence grade II, III, or IV). ${ }^{13)}$

\section{Statistical Analysis}

Multivariate linear regression analysis was applied to evaluate the relationship between the VAS for pain and quantitative variables including tear size, muscle atrophy, patient age, symptom duration, number of torn tendons, and range of motion. The relationship between the VAS for pain and categorical variables (sex, presence of glenohumeral or acromioclavicular joint arthritis, and presence of other comorbidities) was determined by using the chi-squared test. To perform the chi-squared test, the VAS for pain was categorized into three grades $(0-3,4-7,8-10)$. Statistical differences between the VAS for pain according to tear extension were analyzed by the one-way analysis of variance. Statistical analyses were conducted using IBM SPSS (SPSS ver. 
21.0, IBM Corp., Armonk, NY, USA). $p<0.05$ is considered statistically significant.

\section{Results}

\section{Patient Cohort}

Of the 1,379 patients enrolled, 745 patients with a painful rotator cuff tear met the inclusion criteria of this study. There were 312 male patients and 433 female patients. The mean patient age was 61.6 years (range, 41-85 years). Table 1 shows the enrolled patient demographics. Totally, 409 patients had partialthickness rotator cuff tears: 149 patients with articular-side partial-thickness tear, and 260 patients with bursal-side partialthickness tear. Full-thickness rotator cuff tears were detected in 336 patients, and further classified as 69 small-sized tears, 94 medium-sized tears, 81 large-sized tears, and 92 massive-sized tears.

\section{Factors Associated with Pain}

The multivariate linear regression analysis for quantitative variables revealed that patient age was negatively associated with the VAS for pain $(p=0.01)$. Tear characteristics, including tear size $(p=0.53)$, muscle atrophy $(p=0.16)$ and number of torn tendons $(p=0.34)$, were not associated with the VAS for pain. Symptom duration $(p=0.60)$ and passive range of motion (forward flexion: $p=0.30$, external rotation in $90^{\circ}$ abduction: $p=0.18$, external rotation in $0^{\circ}$ abduction: $p=0.19$, internal rotation at the back: $p=0.80$ ) did not correlate with the VAS for pain either (Table 2).

The chi-squared test for categorical variables showed that male sex $(p=0.01)$ and presence of diabetes mellitus $(p<0.001)$ correlated with greater pain. The presence of glenohumeral joint $(p=0.86)$ or acromioclavicular joint arthritis $(p=0.60)$, presence of hypertension $(p=0.63)$, and thyroid disease $(p=0.38)$ did not correlated with the VAS for pain (Table 3). Based on tear extension, patients with bursal-side partial-thickness tear and smallsized full-thickness tears experienced more pain than patients with articular-side partial-thickness tear or medium, large, and massive tears; however, there were no statistically significant differences $(p=0.16)$ (Fig. 2).

\section{Discussion}

This retrospective study demonstrates that shoulder pain is associated with young age, male sex, and the presence of diabetes mellitus. Tear size, muscle atrophy and the number of torn tendons did not correlate with shoulder pain.

There are many treatment modalities for patients with rotator cuff tears, including non-steroidal anti-inflammatory drugs (NSAIDs), intra-articular steroid injection, physical therapy, and surgical management. To decide the treatment modalities, not
Table 1. Patient and Tear Demographics

\begin{tabular}{|c|c|}
\hline Variable & No. of patients \\
\hline \multicolumn{2}{|l|}{ Sex } \\
\hline Male & $312(41.9)$ \\
\hline Female & $433(58.1)$ \\
\hline Mean age (range), yr & $61.6(41-85)$ \\
\hline \multicolumn{2}{|l|}{ VAS for pain } \\
\hline $0-3$ & $234(31.4)$ \\
\hline $4-7$ & $430(57.7)$ \\
\hline $8-10$ & $81(10.9)$ \\
\hline Duration of symptoms, mo & $25.5 \pm 41.8$ \\
\hline \multicolumn{2}{|l|}{ Tear extension } \\
\hline Articular-side partial-thickness tear & $149(20.0)$ \\
\hline Bursal-side partial-thickness tear & $260(34.9)$ \\
\hline Small-sized tear & $69(9.3)$ \\
\hline Medium-sized tear & $94(12.6)$ \\
\hline Large-sized tear & $81(10.9)$ \\
\hline Massive-sized tear & $92(12.3)$ \\
\hline \multicolumn{2}{|l|}{ Muscle atrophy (Goutallier's classification) } \\
\hline Grade 0 & $198(26.6)$ \\
\hline Grade I & $199(26.7)$ \\
\hline Grade II & $245(32.9)$ \\
\hline Grade III & $62(8.3)$ \\
\hline Grade IV & $41(5.5)$ \\
\hline \multicolumn{2}{|l|}{ Glenohumeral joint arthritis } \\
\hline Yes & $41(5.5)$ \\
\hline No & $704(94.5)$ \\
\hline \multicolumn{2}{|l|}{ Acromioclavicular joint arthritis } \\
\hline Yes & $335(45.0)$ \\
\hline No & $410(55.0)$ \\
\hline \multicolumn{2}{|l|}{ Range of motion } \\
\hline Forward flexion (scapular fixed) & $141.3 \pm 14.1$ \\
\hline External rotation in $90^{\circ}$ abduction & $80.7 \pm 16.4$ \\
\hline External rotation in $0^{\circ}$ abduction & $83.8 \pm 13.9$ \\
\hline Internal rotation at the back & $72.1 \pm 22.7$ \\
\hline \multicolumn{2}{|l|}{ Presence of comorbidity } \\
\hline Diabetes mellitus & $121(16.2)$ \\
\hline Hypertension & $211(28.3)$ \\
\hline Thyroid disease & $69(9.3)$ \\
\hline
\end{tabular}

Values are presented as number (\%) or mean \pm standard deviation.

VAS: visual analogue scale. 
Table 2. Multivariate Regression Analysis of the Effect of Quantitative Variables on Visual Analogue Scale for Pain

\begin{tabular}{|lcc|}
\hline \multicolumn{1}{|c}{ Variable } & Regression coefficient & $p$-value \\
\hline Age & -0.11 & $0.01^{*}$ \\
\hline Tear size & -0.03 & 0.53 \\
\hline Muscle atrophy & -0.06 & 0.16 \\
\hline Number of torn tendons & -0.04 & 0.34 \\
\hline Symptom duration & -0.02 & 0.60 \\
\hline Range of motion & & \\
\hline Forward flexion & -0.04 & 0.30 \\
\hline External rotation in $90^{\circ}$ abduction & -0.06 & 0.18 \\
\hline External rotation in $0^{\circ}$ abduction & -0.06 & 0.19 \\
\hline Internal rotation at the back & 0.01 & 0.80 \\
\hline
\end{tabular}

${ }^{*}$ A significant association with $p<0.05$.

Table 3. Chi-squared Test for the Association of Categorical Variables with Visual Analogue Scale for Pain

\begin{tabular}{lc}
\hline \multicolumn{1}{c}{ Variable } & $p$-value \\
\hline Sex & $0.01^{*}$ \\
\hline Glenohumeral joint arthritis & 0.86 \\
\hline Acromioclavicular joint arthritis & 0.60 \\
\hline Diabetes mellitus & $<0.001^{*}$ \\
Hypertension & 0.63 \\
\hline Thyroid disease & 0.38 \\
\hline
\end{tabular}

${ }^{*}$ A significant association with $p<0.05$.

only tear size or extension but also patient symptoms (such as subjective pain) are important. Even in cases of full-thickness rotator cuff tears, conservative treatment is sometimes indicated because of little pain and disability. ${ }^{3,4)}$ Therefore, in order to control shoulder pain, it is imperative to know the risk factors related with preoperative shoulder pain in patients with rotator cuff tears, in the event of factors being correctable. In the present study, young age, male sex and the presence of diabetes mellitus are associated with preoperative shoulder pain. Additional treatment to correct these risk factors, such as controlling the blood glucose level, could exert a positive effect on reducing shoulder pain, although further studies are required.

There is little evidence regarding correlation between age and degree of preoperative pain in rotator cuff tears. ${ }^{5)}$ Postoperatively, increasing age of rotator cuff tear patients is correlated with higher re-tear rates and advanced degrees of pain after tendon repair. ${ }^{14,15)}$ Boileau et al. ${ }^{14)}$ reported that patients over the age of 65 years have a significantly higher re-tear rate after rotator cuff repair. Furthermore, healing failure of the repaired tendon usually correlated with low functional outcomes and advanced degrees of pain. ${ }^{14,15)}$ However, pathogenesis of postoperative pain

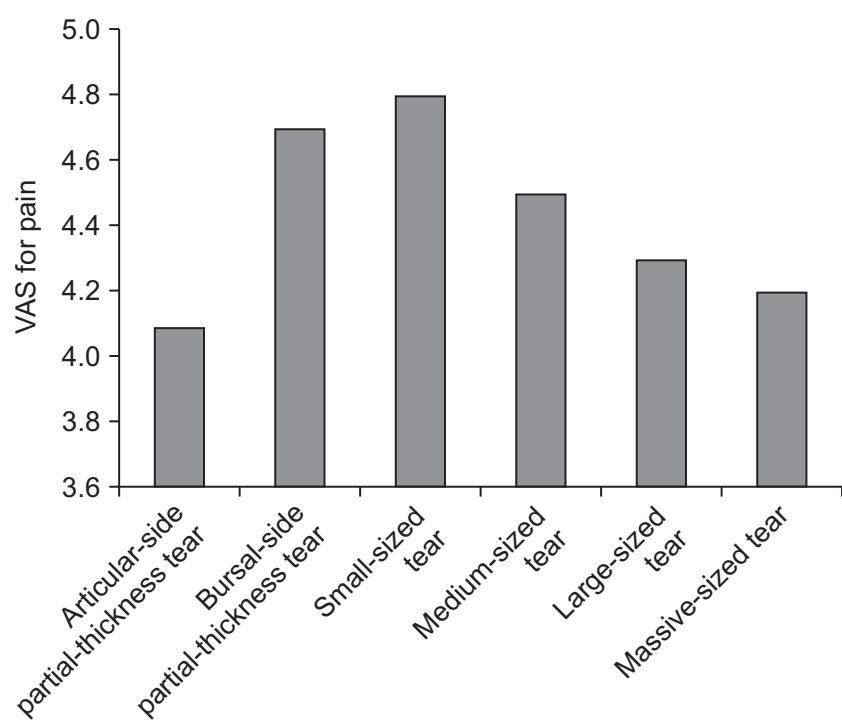

Fig. 2. Visual analogue scale (VAS) for pain according to tear extension. Patients with bursal-side partial-thickness tear and small-sized tear had more pain than patients with articular-side partial-thickness tear or medium-, large-, and massive-sized tears, but these differences were not significant.

development completely differs from preoperative pain conditions. In the present study, elderly patients had less shoulder pain than younger patients at the preoperative stage. This may be explained by the fact that elderly patients generally perform less demanding physical activity than younger patients. However, to quantify the individual physical activity and demands of a patient, further detailed questionnaires are required.

Considering sex of the patient, many clinical studies have demonstrated that females with rotator cuff tear or adhesive capsulitis experience greater shoulder pain as compared to males. ${ }^{16,17)}$ According to a previous study, this difference could be derived from the C-fibers processing method that makes central sensitization greater in female than in male. ${ }^{17)}$ Contrarily, in the present study, males described more severe shoulder pain when compared to females. This result differs from previous studies because of different inclusion and exclusion criteria. The current study attempted to include all patients with painful rotator cuff tear; hence, patients with glenohumeral or acromioclavicular joint arthritis were not excluded, if they presented with a symptomatic rotator cuff tear. Since development or progression of arthritis could be affected by the rotator cuff tear itself, the authors evaluated arthritis as one of the possible factors correlated with shoulder pain in patients with rotator cuff tear. A previous study had also demonstrated that shoulder pain in male and female are affected by different biomechanical and psychosocial factors; shoulder pain of males was associated with high-perceived physical exertion, whereas female shoulder pain was associated with repetitiveness of tasks. ${ }^{18)}$ However, further studies are required to better comprehend the greater pain experienced in males with rotator cuff disease. 
Patients with diabetes mellitus develop several musculoskeletal symptoms that are related to fibrosing conditions. ${ }^{19)}$ The prevalence of adhesive capsulitis, tenosynovitis and osteoporosis are higher in diabetic patients as compared to control. ${ }^{19)}$ Fibrosing conditions in diabetic patients develop through abnormal collagen deposition in the periarticular connective tissue, and abnormal cross-linking of collagen fibers. ${ }^{19)}$ Although additional studies are required, fibrosing conditions and increased proinflammatory cytokines in diabetic patients aggravate pain in rotator cuff tear patients with diabetes mellitus. Clement et al. ${ }^{20)}$ compared the outcomes of arthroscopic rotator cuff repair by considering comorbidity of diabetes mellitus (32 diabetic patients and 32 matched control). They concluded that patients with diabetes show a significant decrease in pain after surgery, but less than their non-diabetic counterparts. This is consistent with our results, which showed a positive correlation between pain and the presence of diabetes mellitus.

In the current study, tear characteristics including tear size, muscle atrophy and number of torn tendons, were not associated with degree of shoulder pain. Several previous studies agreed that tear size is not associated with pain. ${ }^{1,5,9)}$ Subacromial bursitis, rather than a rotator cuff tear itself, is a major factor that results in symptomatic pain in rotator cuff tear patients. ${ }^{21,22)}$ In the subacromial bursa of patients with rotator cuff tears, proinflammatory cytokines such as interleukin-1, interleukin-6, tumor necrosis factor- $\alpha$, stromal cell-derived factor- 1 , and metalloproteases are usually expressed at high levels. ${ }^{21,23)}$ These proinflammatory cytokines mediate the catabolism of collagen and pain generation through cyclo-oxygenase-2 expression. ${ }^{24)}$ In their immunohistochemistry study, Kim et al. ${ }^{21)}$ confirmed that expression of stromal cell-derived factor-1, which is involved in angiogenesis and inflammation, is significantly elevated in the subacromial bursa with rotator cuff disease, and this cytokine is downregulated by cyclo-oxygenase-2 inhibitors and steroids.

Although there were no statistically significant differences in our study, patients with bursal-side partial-thickness tears and small-sized full-thickness tears experienced more pain than patients with articular-side partial-thickness tear, or medium-sized, large-sized or massive-sized tears. Among the full-thickness rotator cuff tears, the tear size was inversely correlated with pain score. Previous studies also demonstrated that patients with partial-thickness rotator cuff tears experienced more pain than patients with full-thickness tears, and this was explained by bursitis, neoinnervation and tension concentration in the remaining tendon. ${ }^{10,11,25)}$ Sano et al. ${ }^{26)}$ conducted a two-dimensional finite element model to evaluate the stress distribution of rotator cuff tendon, and they demonstrated that the partial-thickness rotator cuff tear model showed a high stress concentration around the articular side of footprint. Using ultrasound speckles tracking imaging study, Kim et al. ${ }^{27)}$ evaluated the intra-tendinous strain of a normal supraspinatus tendon, and demonstrated the strain mismatch and shear force between the articular and bursal layers of the supraspinatus tendon. Xu et al. ${ }^{25)}$ designed an immunohistochemistry study of the tendinopathic human rotator cuff tendons, and concluded that new formation of nerve fibers and blood vessels, which might be the source of pain, was more frequently found in early tendinopathic tendons compared with fully torn tendons. Therefore, they emphasized that pain was likely correlated with bursitis or neoinnervation during the early stage, rather than tear size progression.

In the current study, the range of motion was not associated with the VAS for pain. However, stiffness of the shoulder joint usually develops into symptomatic pain. ${ }^{28)}$ This may be explained by the fact that there was only a small number of patients with a significantly limited range of motion in the present study. Only 30 patients had at least a $50 \%$ loss of passive range of motion. In literature, less than $20 \%$ of patients had a rotator cuff tear with significantly limited range of motion. ${ }^{29)}$ Tauro $^{29)}$ demonstrated that only 6 out of 72 patients with rotator cuff tears had a significant deficit in passive motion; of these, only 3 patients had evidence of adhesive capsulitis. Moreover, patients with minimal stiffness usually do not feel symptomatic pain if they use their shoulder within the painless range of motion, as they are limited in only passive motion and not active motion.

There are some limitations to the present study. First, the only outcome parameter in this study was the VAS for pain. The authors did not evaluate the functional scores, since our subject of focus was solely pain outcome. Furthermore, for evaluating shoulder pain, we only used a single parameter, the VAS for pain. For improved understanding of shoulder pain, it would be better to evaluate the VAS for pain during both motion and rest. Second, we could not evaluate the degree of subacromial bursitis by MRI. Subacromial bursa thickness exceeding $3 \mathrm{~mm}$ on MRI usually indicates an abnormal amount of subacromial fluid, which should raise suspicion for bursitis or rotator cuff disease. ${ }^{30}$ However, since very few studies have quantified the degree of subacromial bursitis by MRI, it was difficult to correlate pain and subacromial bursitis by MRI. Lastly, we evaluated the patient medical history with a simple questionnaire. We therefore were unable to quantify the degree of medical comorbidities, especially diabetes mellitus, by blood chemistry measurements of blood glucose and hemoglobin A1C. Uncontrolled diabetes mellitus might have additional effects on symptoms of shoulder pain

\section{Conclusion}

Taken together, our data reveals that shoulder pain is associated with young age, male sex, and the presence of diabetes mellitus in patients with rotator cuff tears. Anatomic measurements of rotator cuff tear characteristics and passive range of motion did not correlate with shoulder pain. We propose that a 
combined treatment regimen for pain management and risk factor correction would be helpful to control preoperative shoulder pain.

\section{References}

1. Dunn WR, Kuhn JE, Sanders R, et al. Symptoms of pain do not correlate with rotator cuff tear severity: a cross-sectional study of 393 patients with a symptomatic atraumatic full-thickness rotator cuff tear. J Bone Joint Surg Am. 2014;96(10):793-800. doi: 10.2106/JBJS.L.01304.

2. Jeong J, Shin DC, Kim TH, Kim K. Prevalence of asymptomatic rotator cuff tear and their related factors in the Korean population. J Shoulder Elbow Surg. 2017;26(1):30-5. doi: 10.1016/ j.jse.2016.05.003.

3. Kijima H, Minagawa H, Nishi T, Kikuchi K, Shimada Y. Longterm follow-up of cases of rotator cuff tear treated conservatively. J Shoulder Elbow Surg. 2012;21(4):491-4. doi: 10.1016/ j.jse.2011.10.012.

4. Petri M, Ettinger M, Brand S, Stuebig T, Krettek C, Omar M. Non-operative management of rotator cuff tears. Open Orthop J. 2016;10:349-56. doi: 10.2174/1874325001610010349.

5. Harris JD, Pedroza A, Jones GL; MOON (Multicenter Orthopedic Outcomes Network) Shoulder Group. Predictors of pain and function in patients with symptomatic, atraumatic full-thickness rotator cuff tears: a time-zero analysis of a prospective patient cohort enrolled in a structured physical therapy program. Am J Sports Med. 2012;40(2):359-66. doi: 10.1177/0363546511426003.

6. Neogi T, Felson D, Niu J, et al. Association between radiographic features of knee osteoarthritis and pain: results from two cohort studies. BMJ. 2009;339:b2844. doi: 10.1136/bmj. b2844.

7. Sadosky AB, Bushmakin AG, Cappelleri JC, Lionberger DR. Relationship between patient-reported disease severity in osteoarthritis and self-reported pain, function and work productivity. Arthritis Res Ther. 2010;12(4):R162. doi: 10.1186/ ar3121.

8. Yamaguchi K, Ditsios K, Middleton WD, Hildebolt CF, Galatz LM, Teefey SA. The demographic and morphological features of rotator cuff disease. A comparison of asymptomatic and symptomatic shoulders. J Bone Joint Surg Am. 2006;88(8):1699-704. doi: 10.2106/JBJS.E.00835.

9. Wylie JD, Suter T, Potter MQ, Granger EK, Tashjian RZ. Mental health has a stronger association with patient-reported shoulder pain and function than tear size in patients with full-thickness rotator cuff tears. J Bone Joint Surg Am. 2016;98(4):2516. doi: 10.2106/JBS.O.00444.

10. Fukuda H. Partial-thickness rotator cuff tears: a modern view on Codman's classic. J Shoulder Elbow Surg. 2000;9(2):163-8. doi: 10.1067/mse.2000.101959.
11. Fukuda $\mathrm{H}$. The management of partial-thickness tears of the rotator cuff. J Bone Joint Surg Br. 2003;85(1):3-11.

12. Goutallier D, Postel JM, Bernageau J, Lavau L, Voisin MC. Fatty muscle degeneration in cuff ruptures. Pre- and postoperative evaluation by CT scan. Clin Orthop Relat Res. 1994;(304):7883.

13. Kellgren JH, Lawrence JS. Radiological assessment of osteoarthrosis. Ann Rheum Dis. 1957;16(4):494-502. doi: 10.1136/ ard.16.4.494.

14. Boileau P, Brassart N, Watkinson DJ, Carles M, Hatzidakis AM, Krishnan SG. Arthroscopic repair of full-thickness tears of the supraspinatus: does the tendon really heal? J Bone Joint Surg Am. 2005;87(6):1229-40. doi: 10.2106/JBJS.D.02035.

15. Downie BK, Miller BS. Treatment of rotator cuff tears in older individuals: a systematic review. J Shoulder Elbow Surg. 2012;21(9):1255-61. doi: 10.1016/j.jse.2011.11.019.

16. Gumina S, Candela V, Passaretti D, et al. Intensity and distribution of shoulder pain in patients with different sized postero-superior rotator cuff tears. J Shoulder Elbow Surg. 2014;23(6):807-13. doi: 10.1016/j.jse.2013.09.011.

17. Kindler LL, Valencia C, Fillingim RB, George SZ. Sex differences in experimental and clinical pain sensitivity for patients with shoulder pain. Eur J Pain. 2011;15(2):118-23. doi: 10.1016/ j.ejpain.2010.06.001.

18. Bodin J, Ha C, Chastang JF, et al. Comparison of risk factors for shoulder pain and rotator cuff syndrome in the working population. Am J Ind Med. 2012;55(7):605-15. doi: 10.1002/ ajim.22002.

19. Merashli M, Chowdhury TA, Jawad AS. Musculoskeletal manifestations of diabetes mellitus. QJM. 2015;108(11):853-7. doi: 10.1093/qjmed/hcr106.

20. Clement ND, Hallett A, MacDonald D, Howie C, McBirnie J. Does diabetes affect outcome after arthroscopic repair of the rotator cuff? J Bone Joint Surg Br. 2010;92(8):1112-7. doi: 10.1302/0301-620X.92B8.23571.

21. Kim YS, Bigliani LU, Fujisawa M, et al. Stromal cell-derived factor 1 (SDF-1, CXCL12) is increased in subacromial bursitis and downregulated by steroid and nonsteroidal anti-inflammatory agents. J Orthop Res. 2006;24(8):1756-64. doi: 10.1002/ jor.20197.

22. Gotoh M, Hamada K, Yamakawa H, Inoue A, Fukuda H. Increased substance $\mathrm{P}$ in subacromial bursa and shoulder pain in rotator cuff diseases. J Orthop Res. 1998;16(5):618-21. doi: 10.1002/jor.1100160515.

23. Voloshin I, Gelinas J, Maloney MD, O'Keefe RJ, Bigliani LU, Blaine TA. Proinflammatory cytokines and metalloproteases are expressed in the subacromial bursa in patients with rotator cuff disease. Arthroscopy. 2005;21(9):1076.e1-9. doi: 10.1016/j.arthro.2005.05.017.

24. Crofford LJ. COX-1 and COX-2 tissue expression: implications and predictions. J Rheumatol Suppl. 1997;49:15-9. 
25. $\mathrm{Xu} \mathrm{Y,} \mathrm{Bonar} \mathrm{F,} \mathrm{Murrell} \mathrm{GA.} \mathrm{Neoinnervation} \mathrm{in} \mathrm{rotator} \mathrm{cuff} \mathrm{ten-}$ dinopathy. Sports Med Arthrosc Rev. 2011;19(4):354-9. doi: 10.1097/JSA.0b013e318229d7e3.

26. Sano H, Wakabayashi I, Itoi E. Stress distribution in the supraspinatus tendon with partial-thickness tears: an analysis using two-dimensional finite element model. J Shoulder Elbow Surg. 2006;15(1):100-5. doi: 10.1016/j.jse.2005.04.003.

27. Kim YS, Kim JM, Bigliani LU, Kim HJ, Jung HW. In vivo strain analysis of the intact supraspinatus tendon by ultrasound speckles tracking imaging. J Orthop Res. 2011;29(12):1931-7. doi: 10.1002/jor.21470.

28. Tauro JC, Paulson M. Shoulder stiffness. Arthroscopy. 2008;24(8):949-55. doi: 10.1016/j.arthro.2008.03.014.

29. Tauro JC. Stiffness and rotator cuff tears: incidence, arthroscopic findings, and treatment results. Arthroscopy. 2006;22(6):581-6. doi: 10.1016/j.arthro.2006.03.004.

30. White EA, Schweitzer ME, Haims AH. Range of normal and abnormal subacromial/subdeltoid bursa fluid. J Comput Assist Tomogr. 2006;30(2):316-20. 